\title{
A longitudinal study of Ambulatory Physician Encounters, Emergency Room visits and Hospitalizations by patients with Rheumatoid Arthritis
}

\section{A 13 year population health study}

\author{
John G Hanly, Kara Thompson, Chris Skedgel
}

\begin{abstract}
Objective: To determine total physician encounters, emergency room (ER) visits and hospitalizations in an incident cohort of rheumatoid arthritis (RA) cases and matched control patients over 13 years.

Methods: A retrospective cohort study was performed utilizing administrative health care data from approximately 1 million people with access to universal healthcare. Using ICD-9 and ICD-10 diagnostic codes, 7 RA case definitions were used. Each case was matched by age and sex to 4 randomly selected controls. Data included physician billings, ER visits and hospital discharges over 13 years.
\end{abstract}

Results: The number of incident RA cases varied from 3,497 to 27,694 depending upon the case definition. The mean age varied from 54.3 to 65.0 years and the proportion of females from 67.8 to 71.3\%. Physician encounters by RA patients was significantly higher than controls, was highest in the index year, declining promptly thereafter for all case definitions and by $12.2-46.8 \%$ after 10 years. Encounters with subspecialty physicians fell by $61 \%$ (Rheumatologists) and 34\% (Internal medicine). 
In contrast, clinical encounters with family physicians and other physicians fell by only $9 \%$. Visits to the ER and hospital admissions were also significantly higher in RA cases, particularily early in the disease and fell significantly over the follow-up.

Conclusion: In RA patients, health care utilization is highest in the first year following the diagnosis which is also the time of maximal involvement by rheumatologists. Utilization declines over time and encounters with patient's family physicians predominate over other physician groups.

Key indexing terms: RA, Health care utilization

Division of Rheumatology, Department of Medicine and Department of Pathology, Queen Elizabeth II Health Sciences Centre and Dalhousie University, Halifax, Nova Scotia, Canada;

Department of Medicine and Research Methods Unit, Queen Elizabeth II Health Sciences Centre and Dalhousie University, Halifax, Nova Scotia, Canada;

Atlantic Clinical Cancer Research Unit, Capital Health, Nova Scotia, Canada.

Health Economics Group, Norwich Medical School, University of East Anglia, United Kingdom.

$\begin{array}{lll}\text { JG Hanly } & \text { Rheumatologist } & \text { MD, FRCPC } \\ \text { K Thompson } & \text { Biostatistician } & \text { MSc } \\ \text { C Skedgel } & \text { Health Economist } & \text { PhD }\end{array}$


Corresponding author: Dr. John G Hanly, Division of Rheumatology, Nova Scotia Rehabilitation Centre (2 ${ }^{\text {nd }}$ Floor), 1341 Summer Street, Halifax, Nova Scotia, Canada, B3H 4K4.

Telephone: (902) 473 3818; Fax: (902) 473 7019; Email: john.hanly@cdha.nshealth.ca

Word count (text only, not including abstract): 3,422

Word count (abstract): 245

Running title: Health care utilization in RA 
Rheumatoid arthritis (RA) is the most frequent chronic autoimmune inflammatory rheumatic diseases, with a higher frequency in women. Although it has the potential to affect any organ system in the body, the predominant manifestations are in the musculoskeletal system. This inflammatory arthropathy runs a variable and unpredictable course but usually leads to significant morbidity and premature mortality if left untreated. The clinical outcome for both mild and severe cases is improved by a prompt and accurate diagnosis, appropriate access to health care providers and institution of evidence based treatments.

Planning for the provision of future health resources required for the diagnosis and treatment of RA patients starts with an assessment of previous and current resource utilization. Different research methodologies have been used to address this, including case-control strategies in tertiary referral centers (1), observational cohorts $(2,3)$ and mining of population health administrative datasets (4-6). Resource utilization and associated costs have been examined in various geographic locations including the USA $(1,7)$, Canada $(5,8)$ and other countries $(2,9)$. This is appropriate as findings are influenced by health care system delivery which is highly variable. Due to the rapid pace of reform of health care delivery and the chronicity of RA which extends over a patient's lifetime, it is necessary to periodically update utilization in representative geographic locations. As part of an overall evaluation of health care resource utilization by RA patients in our region we examined the clinical encounters with primary care and subspecialty physicians, emergency room visits and hospitalizations in RA and control patients over 13 years using a validated population health dataset.

\section{Materials and Methods}

Study populations and controls: This was a retrospective cohort study of patients with a diagnosis of RA within the Nova Scotia Medical Services Insurance (MSI) program. Nova Scotia is a Canadian 
province of approximately 1 million inhabitants. There are 3,500 physicians in Nova Scotia of which approximately $50 \%$ work in primary care, $7 \%$ are general internists and $0.3 \%$ are adult rheumatologists. Health care services including acute and elective hospitalizations and ambulatory physician visits are universally provided as specified under the Canada Health Act. The eligible population for the study was Nova Scotia residents who were enrolled in the MSI program between April $1^{\text {st }} 1997$ and March $31^{\text {st }}$ 2011. This excludes First Nation Canadians and members of the Canadian armed forces. Incident cases of RA were defined as those without a physician billing for the same diagnosis in the 5 years preceeding 1997 (5). Prevalent cases included both incident and nonincident cases. Patients with RA were matched one to four by age and sex to a control cohort of patients who were also enrolled in the MSI program at the time of their matched case's date of diagnosis and who never had a diagnosis of RA or other connective tissue disease.

The data was obtained from existing databases accessed through the Population Health Research Unit (PHRU) (now Health Data Nova Scotia (HDNS)) in the Department of Community Health \& Epidemiology at Dalhousie University in Halifax, Nova Scotia, Canada. Within this unit there are secure research computing facilities on site and access to data is governed by PHRU/HDNS Data Access Guidelines and Procedures. Electronic utilization data from the Nova Scotia Senior Pharmacare Program (NSSPP) for seniors (age $\geq 65$ ), the Canadian Institute of Health Information (CIHI) Hospital Discharge Abstracts database and the MSI Physician Billings database were linked via medical services insurance (MSI) number. The study protocol was reviewed and approved by the Capital Health (now Nova Scotia Health Authority, central zone) Research Ethics Board (CDHARS/2010-118). Informed consent from individual patients was not required as the study utilized secondary administrative data. 
Case definitions for identification of RA cases and validation: The following 7 individual case definitions, 3 of which are based upon previously published work, were used to identify cases of RA in the administrative databases. We have previously validated these case definitions against a clinical dataset of RA patients and controls (10). The diversity of the decision rules provides a range of sensitivity and specificity for case ascertainment, thus permitting a sensitivity analysis of health care utilization.

1 Any encounter: Any single diagnostic code for RA by a physician.

\#2 MacLean (11): Two physician visits for RA at least 2 months apart.

\#3 MacLean/Lacaille (5): MacLean-like algorithm with Lacaille variation (excluded individuals with at least 2 visits, at least 2 months apart, subsequent to the second RA visit, with 2 identical diagnoses of other inflammatory arthritides and connective tissue diseases (psoriatic arthritis, ankylosing spondylitis, and other spondylarthropathies, systemic lupus erythematosus (SLE), scleroderma, Sjogren's syndrome, dermatomyostis, polymyositis, other connective tissue diseases, primary systemic vasculitis) and those where a diagnosis of RA by a non-rheumatologist was not confirmed if/when the individual saw a rheumatologist.

\#4 Shipton-like: Three RA diagnostic billing codes, over any time period, rather than in 3 consecutive years as described by Shipton et al (12).

\#5 Hospitalization: At least one hospitalization where RA was in the diagnostic codes.

\#6 Rheumatologist: At least one RA code contributed by a rheumatologist. 
\#7 Combination: MacLean-like algorithm (2 non-Rheumatology physician visits for RA at least 2 months apart, within a 2 year period) or at least one RA code contributed by a rheumatologist or at least one hospitalization where RA was in the diagnostic codes and Lacaille variation, i.e. excluding individuals with at least 2 visits, at least 2 months apart, subsequent to the second visit, with 2 identical diagnoses of other inflammatory arthritides and connective tissue diseases (psoriatic arthritis, ankylosing spondylitis, and other spondylarthropathies, SLE, scleroderma, Sjogren's syndrome, dermatomyostis, polymyositis, other connective tissue diseases, primary systemic vasculitis) and excluding those where a diagnosis of RA by a non-rheumatologist was not confirmed if/when the individual saw a rheumatologist.

Data collection: Individual level data were obtained. Computerized claims were linked by encrypted health card number to the Canadian Institute of Health Information (CIHI) Hospital Discharge Abstracts and MSI Physician Billings for fiscal years from April $1^{\text {st }} 1997$ and March 31 2011.

The following ICD-9 and ICD-10 diagnostic codes were used:

Rheumatoid arthritis (ICD-9: 714.0, 714.1, 714.2. ICD-10: MO5 - MO5.9, MO6.0, MO6.8, MO6.9).

Psoriatic arthritis (ICD-9: 696.0. ICD-10: L40.5)

Ankylosing spondylitis (ICD-9: 720.0. ICD-10: M45)

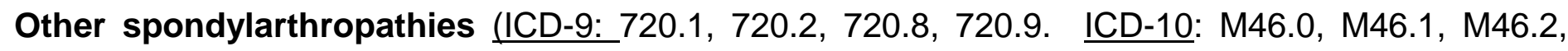
M46.3, M46.4, M46.5 M46.8, M46.9)

Systemic lupus erythematosus (ICD-9: 710.0. ICD-10: ICD-10: M 32, M32.1, M32.8, M32.9).

Scleroderma (ICD-9: 710.1. ICD-10: M34)

Sjogren's syndrome (ICD-9: 710.2. ICD-10: M35.0)

Dermatomyositis (ICD-9: 710.3. ICD-10: M33.1, M33.9) 
Polymyositis (ICD-9: 710.4. ICD-10: M33.2)

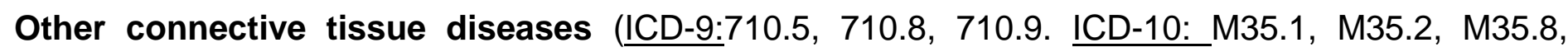
M35.9)

Primary systemic vasculitis: $\quad(\underline{I C D}-9: \quad 446.0,446.2,446.4,446.5, \quad 446.7, \quad 447.6$. ICD-10: D69.0, M31.0, M30.0, M31.3, M31.4, M31.5, M31.6, M31.7, M31.8, M31.9).

Using chapter sections from ICD manuals selected co-morbidities over the study period were expressed as a proportion of affected RA cases and controls using the following ICD-9 and ICD-10 diagnostic codes:

Cancer: all malignancies except lymphoma (ICD-9: 140-208, excluding 200-203. ICD-10: C00D48, excluding C81-C85;

Coronary heart disease (CHD) (ICD-9: 410-414. ICD-10: I20-I25)

Cardiovascular disease excluding CHD (ICD-9: 390-459 excluding 410-414. ICD-10: II00-I99 excluding I20-I25)

Diabetes (ICD-9: 250. ICD-10: E10-E14)

Infection (ICD-9: 001-139, 465, 480-488. ICD-10: A00-B99, J06,J10-J18)

Lymphoma (ICD-9: 200-203. ICD-10: C81-C85)

Mental health (ICD-9: 290-319. ICD-10: F00-F99)

Renal impairment (ICD-9: 580-589. ICD-10: N00-N19)

Statistical analysis: The data were analyzed with SAS software v8.3 and SAS/Stat software 12.1 v9.3 (SAS Institute Inc., Cary N.C., USA). Descriptive statistics were used to characterize the RA and control cohorts and variables included age, sex, number of ambulatory visits, number of emergency 
room visits, number of hospitalizations, and diagnosing physician groups. Censorship of data was addressed by using patient year exposures which was defined by each individual's utilization or eligibility for utilization in number of years between their incident diagnosis or matching and the last contact with the health service (i.e. physician contact, emergency room visit and hospitalization). Linear regression and negative binomial models at the aggregate data level were run to examine differences in utilization between cases and controls and utilization over time. Models were also adjusted for the interaction of index year of utilization and case/control group. The log of patient year exposure was used as the offset in negative binomial models. The relationship between individual comorbidities and case definition group was examined using the chi-square test for independence. The analysis was run separately for each case definition. Finally, multivariate models were run to investigate the relative effects of covariates on utilization measures. Age, sex, year of diagnosis, comorbidities and access to care as measured by rural residence were modeled. Rural was identified as having a residence postal code with a zero as the second character. All observations for each year were pooled with a dummy included to distinguish cases versus controls. This analysis was run for the combination case definition (\#7) only.

\section{Results}

Patients and controls: The number of RA cases identified in the administrative datasets and available for study over the 13 years of observation varied with the 7 case definitions used (Table 1). As previously described (13) the definition with the greatest sensitivity was \#1 (a single encounter with any physician) and the most specific definition was \#5 (hospitalization for which RA was listed as one of the diagnostic codes). Using these two definitions the number of incident cases of RA varied from 3,497 to 27,694 and the number of prevalent cases varied from 15,328 to 81,866 over the period 
of study. Cases had a comparable mean age and sex distribution regardless of the case definition for RA patients with the exception of patients identified through a hospital admission (case definition \#5) (Table 1) who were older. There was no significant difference between RA cases and controls in the number of patient year exposures.

Physician consultations: Starting from the index year (i.e. year of diagnosis of RA in this incident cohort) the number of physician encounters per patient per year for cases and controls over the duration of followup is illustrated in Figure 1. RA cases were identified using the 7 case definitions for RA. Utilization reflects the combination of outpatient ambulatory assessments and inpatient consultations by family physicians, general internists, rheumatologists and other physician groups. For all case definitions there was significantly higher utilization by RA cases compared to controls. $(p<0.001)$. In RA cases the utilization was highest in the index year and fell thereafter. There was a significant fall in utilization by RA cases over time $(p<0.001)$ with all case definitions. For four RA case definitions the utilization curves dipped substantially during the last 3 years of observation. The same observation occurred for the matched control group which suggests that the cause was unrelated to RA.

The breakdown in physician encounters by specialty group and the change over time is illustrated in Figure 2 using RA case definition \#7 (Combination). For all case definitions the utilization of all physician groups was significantly higher for RA cases than for their matched controls $(P<0.01)$. This was apparent both at the initial encounter and over time. There was a significant fall in the utilization of all physician groups by RA cases over time $(p<0.001)$ with all case definitions with the exception of utilization of other physician groups using case definition \#1. Subspecialty encounters was highest early in the disease course and by the fourth assessment the frequency fell by $61 \%$ 
(Rheumatologists), 34\% (Internal medicine) and 14\% (other physicians). In contrast, the frequency of visits to family physicians only fell by $9 \%$ over the same time and remained high for the duration of the study.

Emergency room visits: Utilization of the emergency room by RA cases and controls is illustrated in Figure 3. Regardless of which case definition for RA that was used, visits to the emergency room were significantly more frequent by RA cases $(P<0.001)$ which was most apparent early in the disease. There was a significant fall in emergency visits by RA cases over time $(p<0.01)$ with all case definitions.

Hospitalizations: Hospital admission rates in RA cases and controls are illustrated in Figure 4. For RA cases, regardless of the definition, the hospital admission rate was significantly higher $(P<0.001)$ compared to controls. This was especially true early in the disease course. There was a significant fall in hospitalizations for RA cases over time $(p<0.001)$ with all case definitions with the exception of case definition \#1 (encounter for RA with any physician). The spikes in hospital admissions seen among the cases most likely reflect random events among the relatively small numbers of patients, especially with some case definitions. Not surprisingly, patients identified through a hospital admission associated with RA (case definition \#5) had a high admission rate over time and the data point for the admission rate at the first encounter was so high (8.17) that it negated the ability to discriminate other hospitalization curves and was not included in the graph.

Comparison of utilization rates between RA cases and controls: In view of the similarity in patterns of utilization, regardless of the case definition, differences in utilization between RA cases and controls were derived for a representative case definition, namely \#7 (Combination). Estimates of utilization by RA cases compared to controls are summarized in Table 2. 
Co-morbidities: The proportions of RA patients and matched controls with selected co-morbidities over the period of study are summarized in Table 1. Regardless of which RA case definition was used, all of the co-morbidities were more frequent in RA cases compared to their matched controls $(p<0.0001)$ with the exception of coronary heart disease $(p=0.346)$ and renal impairment $(p=0.295)$ in RA case definition \#3 (MacLean-like algorithm with Lacaille variation. For the same case definition the higher frequency of diabetes $(p=0.004)$, lymphoma $(p=0.020)$ in RA compared to controls reached a lower level of statistical significance. Within RA cases, patients identified through a hospital admission associated with RA (case definition \#5) had a higher proportion with co-morbidities.

Multivariate analysis indicated that higher physician utilization was associated with female sex, older age, urban dwelling and all of the co-morbidities $(p<0.0001)$ identified. There were similar associations for emergency room visits $(p<0.0001)$ with the exception that these were more likely to occur for males. Hospitalization was associated with male sex, older age, urban dwelling and all of the co-morbidities identified $(p<0.005)$ with the exception of cardiovascular disease, diabetes and mental health.

\section{Discussion}

Health care utilization and associated costs have been studied in RA and other chronic rheumatic diseases using different research methodologies. Most studies have involved secondary use of health administrative data on prevalent cohorts followed over short time frames (14). In our study we wished to examine the change in health care utilization in the total population of RA patients in our geographic area at the time of diagnosis and over the ensuing years and to compare it to that seen in 
population controls. To this end we studied an incident cohort of RA patients followed for up to 13 years using health administrative data which was previously validated against a clinical dataset.

Studies of health care utilization have consistently found that it is higher in RA patients than in comparator groups of patients $(3,15)$. Utilization may be influenced by a variety of factors, including medication costs, higher co-morbidities leading to increased hospitalization (7), and the type of health care delivery system. Previous studies have been cross-sectional, retrospective and longitudinal in design, using prevalent rather than incident RA cases. In the current study, utilization was assessed in the first year that the incident case occurred in the dataset which was taken as the year of diagnosis of RA. Subsequent utilization was tracked for up to 13 years. Seven case definitions for RA with a range of sensitivities and specificities were used in order to capture the full spectrum of RA. The accuracy of these definitions and the identification of incident and prevalent cases of RA have been published in detail elsewhere (10). Their use in this and future studies allows a form of sensitivity analyses for utilization and costs. The cases identified by each definition were matched by age and sex to 4 controls. The trends in health care utilization were remarkably consistent across case definitions. Regardless of the RA case definition, physician encounters were highest in the year of diagnosis, trending lower in subsequent years but always remained above that in matched controls. Although not proven it seems reasonable to assume that the gradual increase in utilization in the controls over time was most likely due to the change in health care needs with increasing age.

It is to be expected that multiple physician groups will be involved in the care of patients with RA given the systemic nature of the illness and the higher frequency of co-morbidities. Indeed, we recently reported a very similar pattern of health care utilization in patients with SLE (16). In the 
current study, physician encounters were categorized into those with family physicians, general internists, rheumatologists and others. Utilization of all 4 physician groups was substantially higher in RA cases compared to controls over the entire study. Encounters with family physicians, who are responsible for delivering primary care in the Canadian health care system, were the most frequent. Subspecialty care was utilized most frequently early in the disease course, falling substantially over the next 3 years. In contrast, the frequency of encounters with family physicians changed relatively little over time. The interpretation and implications of these observations are two-fold. First, access to subspecialty care is most frequent and thus most critical around the time of diagnosis of RA and in the first few years of followup when the disease is being stabilized. Second, the utilization of family physicians remains high but relatively constant over the course of the illness which underlines the important role that family physicians play in the long-term management of RA patients. Given the need to prevent and treat co-morbidities, which were more frequent in RA patients compared to matched controls in our study, the strategic delivery of effective care will require ongoing coordination by rheumatologists and primary care physicians.

The frequency of admissions to hospital is an indicator of the clinical impact of a medical illness and has previously been demonstrated to be higher in patients with RA compared to controls (1). The current study confirms this observation, and well as demonstrating a higher frequency of hospital emergency room visits which is likely linked to hospital admission rates. Furthermore, there was a consistent change in the pattern of utilization over the 13 years of observation. Both services were accessed most frequently in the first year following the diagnosis of RA (index year), and declined thereafter to eventually reach the same frequency as in controls by the end of the study. Although this could reflect a survivor effect due to excess mortality in RA cases early in the disease course, a more likely explanation is that following the diagnosis of RA, appropriate treatment is initiated leading 
to improved disease control and reduced need to visit the emergency room or require admission to hospital.

There are a number of strengths to the current study. First, due to the Canada Health Act all patients accessed health care through a single provider, ensuring comprehensive data capture for all physician encounters, emergency room visits and hospitalizations. Second, the Nova Scotia population is stable with a mix of urban and rural communities and a range of socioeconomic groups and thus represents many of the features of a general Canadian population, albeit with the exception of a diversified racial/ethnic mix. Third, the use of 7 validated case definitions reduces the risk of bias that could arise from using a more limited strategy for identifying cases and controls.

There are also some limitations to the study. First, due to the homogenous nature of the Nova Scotia population it was not possible to examine the effect of race/ethnicity on health care utilization. Second, athough the definition of incident cases was in agreement with traditional methodology in population health studies (5) it would not have excluded RA patients with longstanding disease who relocated to Nova Scotia during the period of study. Third, the cases were not stratified for disease activity or severity, in which utilization patterns may have been different. Fourth, although the presence and impact of several predefined co-morbidities were examined there are other potentially important co-morbidities (e.g. smoking) that were not available for inclusion in the analysis. Finally, comparative data on patients with other chronic disease in a population of similar age and sex was not available. Future studies will address these deficiencies and determine the economic costs of health care utilization in this cohort of RA patients. 


\section{Acknowledgment}

Support for this work was provided by the John \& Marian Quigley Endowment Fund for Rheumatology, and unrestricted funding from Glaxo Smith Kline, Canada and Roche Canada. We

gratefully acknowledge the expertise of Lynn Lethbridge for her assistance in generating reports from the administrative dataset. 


\section{References}

1. Michet CJ, 3rd, Strobova K, Achenbach S, Crowson CS, Matteson EL. Hospitalization rates and utilization among patients with rheumatoid arthritis: a population-based study from 1987 to 2012 in Olmsted County, Minnesota. Mayo Clin Proc. 2015;90(2):176-83.

2. Dale J, Paterson C, Tierney A, Ralston SH, Reid DM, Basu N, et al. The Scottish Early Rheumatoid Arthritis (SERA) Study: an inception cohort and biobank. BMC Musculoskelet Disord. 2016;17(1):461.

3. Lanes SF, Lanza LL, Radensky PW, Yood RA, Meenan RF, Walker AM, et al. Resource utilization and cost of care for rheumatoid arthritis and osteoarthritis in a managed care setting: the importance of drug and surgery costs. Arthritis Rheum. 1997;40(8):1475-81.

4. El-Gabalawy R, Mackenzie CS, Shooshtari S, Sareen J. Comorbid physical health conditions and anxiety disorders: a population-based exploration of prevalence and health outcomes among older adults. Gen Hosp Psychiatry. 2011;33(6):556-64.

5. Lacaille D, Anis AH, Guh DP, Esdaile JM. Gaps in care for rheumatoid arthritis: a population study. Arthritis Rheum. 2005;53(2):241-8.

6. Lix LM, Yogendran MS, Shaw SY, Burchill C, Metge C, Bond R. Populationbased data sources for chronic disease surveillance. Chronic Dis Can. 2008;29(1):31-8. 
7. Han GM, Han XF. Comorbid conditions are associated with healthcare utilization, medical charges and mortality of patients with rheumatoid arthritis. Clin Rheumatol. 2016;35(6):1483-92.

8. Ohinmaa AE, Thanh NX, Barnabe C, Martin L, Russell AS, Barr SG, et al. Canadian estimates of health care utilization costs for rheumatoid arthritis patients with and without therapy with biologic agents. Arthritis Care Res (Hoboken). 2014;66(9):1319-27.

9. Lai NS, Tsai TY, Li CY, Koo M, Yu CL, Lu MC. Increased frequency and costs of ambulatory medical care utilization prior to the diagnosis of rheumatoid arthritis: a national population-based study. Arthritis Care Res (Hoboken). 2014;66(3):3718.

10. Hanly JG, Thompson K, Skedgel C. The use of administrative health care databases to identify patients with rheumatoid arthritis. Open Access Rheumatology: Research and Reviews. 2015;7:69-75.

11. MacLean $\mathrm{CH}$, Louie R, Leake B, McCaffrey DF, Paulus HE, Brook RH, et al. Quality of care for patients with rheumatoid arthritis. JAMA. 2000;284(8):984-92.

12. Shipton D, Glazier RH, Guan J, Badley EM. Effects of use of specialty services on disease-modifying antirheumatic drug use in the treatment of rheumatoid arthritis in an insured elderly population. Med Care. 2004;42(9):907-13.

13. Hanly JG, Thompson K, Skedgel C. The use of administrative health care databases to identify patients with rheumatoid arthritis. Open Access Rheumatol. 2015;7:69-75. 
14. Widdifield J, Labrecque J, Lix L, Paterson JM, Bernatsky S, Tu K, et al. Systematic review and critical appraisal of validation studies to identify rheumatic diseases in health administrative databases. Arthritis Care Res (Hoboken). 2013;65(9):1490-503.

15. Hansagi $\mathrm{H}$, Allebeck $\mathrm{P}$, Allander E. Utilization of hospital care among persons with rheumatoid arthritis compared with controls. A 13-year follow-up of an epidemiological survey. Scand J Rheumatol. 1985;14(4):403-10.

16. Hanly JG, Thompson K, Skedgel C. Utilization of Ambulatory Physician Encounters, Emergency Room Visits, and Hospitalizations by Systemic Lupus Erythematosus Patients: A 13-Year Population Health Study. Arthritis Care Res (Hoboken). 2016;68(8):1128-34. 
1. Lacaille D, Anis AH, Guh DP, Esdaile JM. Gaps in care for rheumatoid arthritis: a population study. Arthritis Rheum 2005;53:241-8.

2. Hanly JG, Thompson K, Skedgel C. The use of administrative health care databases to identify patients with rheumatoid arthritis. Open Access

Rheumatology: Research and Reviews 2015;7:69-75.

3. MacLean $\mathrm{CH}$, Louie R, Leake B, McCaffrey DF, Paulus HE, Brook RH, et al. Quality of care for patients with rheumatoid arthritis JAMA. 2000;284:984-92.

4. Shipton D, Glazier RH, Guan J, Badley EM. Effects of use of specialty services on disease-modifying antirheumatic drug use in the treatment of rheumatoid arthritis in an insured elderly population. Med Care 2004;42:907-13.

5. Hanly JG, Thompson K, Skedgel C. The use of administrative health care databases to identify patients with rheumatoid arthritis. Open Access Rheumatol 2015;7:69-75.

6. Widdifield J, Labrecque J, Lix L, Paterson JM, Bernatsky S, Tu K, et al. Systematic review and critical appraisal of validation studies to identify rheumatic diseases in health administrative databases. Arthritis Care Res (Hoboken) 2013;65:1490-503.

7. Hansagi $\mathrm{H}$, Allebeck $\mathrm{P}$, Allander E. Utilization of hospital care among persons with rheumatoid arthritis compared with controls. A 13-year follow-up of an epidemiological survey. Scand J Rheumatol1985;14:403-10.

8. Lanes SF, Lanza LL, Radensky PW, Yood RA, Meenan RF, Walker AM, et al. Resource utilization and cost of care for rheumatoid arthritis and osteoarthritis in 
a managed care setting: the importance of drug and surgery costs. Arthritis Rheum 1997;40:1475-81.

9. Han GM, Han XF. Comorbid conditions are associated with healthcare utilization, medical charges and mortality of patients with rheumatoid arthritis. Clin Rheumatol 2016;35:1483-92.

10. Michet CJ, 3rd, Strobova K, Achenbach S, Crowson CS, Matteson EL. Hospitalization rates and utilization among patients with rheumatoid arthritis: a population-based study from 1987 to 2012 in Olmsted County, Minnesota. Mayo Clin Proc 2015;90:176-83. 


\section{Legends for figures:}

Figure 1: Total physician encounters per year in RA patients (left panel) and controls (right panel) in the index year and over the following 13 years using 7 definitions for RA to identify cases in administrative datasets. RA cases were matched one to four by age and gender to a control cohort of patients who were enrolled in the same datasets but without a diagnosis of RA or other connective tissue diseases.

Figure 2: Physician encounters per year by specialty group in RA patients (left panel) in the index year and over 13 years of followup using case definition \#7 (Combination) to identify RA cases. These were matched one to four by age and gender to a control cohort of patients (right panel) who were enrolled in the same datasets but without a diagnosis of RA or other connective tissue diseases.

Figure 3: Utilization of the emergency room reflected in visits per year by RA cases (left panel) and controls (right panel) in the index year and over the following 13 years using 7 definitions for RA to identify cases in administrative datasets. RA cases were matched one to four by age and gender to a control cohort of patients who were enrolled in the same datasets but without a diagnosis of RA or other connective tissue diseases.

Figure 4: Hospital admission rates per year by RA cases (left panel) and controls (right panel) in the index year and over the following 13 years using 7 definitions for RA to 
identify cases in administrative datasets. RA cases were matched one to four by age and gender to a control cohort of patients who were enrolled in the same datasets but without a diagnosis of RA or other connective tissue diseases. For patients identified through a hospital admission associated with RA (case definition \#5) the data point for the admission rate at the first encounter was 8.17 and was not included in the graph. 